\title{
"Sus mandamientos no son penosos": un abordaje historiográfico de la relación entre protestantismo y aflicción
}

Recibido: 26/04/2021. Aceptado:13/08/2021.

\begin{abstract}
Resumen
En los estudios históricos sobre el protestantismo existe un topos que consiste en asociar distintas denominaciones, en especial el calvinismo, con la producción de un sufrimiento psicológico profundo y patológico. Aunque el origen de este tópico es antiguo, su persistencia en el ámbito académico deriva en gran medida de la obra de Max Weber. Este artículo ofrece un análisis de ese tema en la historiografía sobre el protestantismo inglés. En primer lugar, describe el derrotero de ese problema historiográfico y presenta las críticas que se realizaron en distintos momentos. En segundo término, da cuenta de los aportes recientes de la historia de las emociones que podrían permitir un enfoque superador. Finalmente, destaca la necesidad de observar la dimensión polémica de los discursos acerca de la aflicción protestante y sostiene que, para estudiarla, es preciso considerar especialmente el contexto de producción y el vocabulario específico de las fuentes primarias.
\end{abstract}

Palabras clave: Max Weber, protestantismo, aflicción, desesperación, melancolía.

"His Commandments Are Not Grievous": A Historiographical Approach of the Relation between Protestantism and Affliction

\begin{abstract}
Within the historical studies on Protestantism, there is a common topos that links different denominations, specifically Calvinism, with the production of a deep and pathological psychological pain. Although the origin of this topic is not new, its persistence in scholarly writing stems largely from the works of Max Weber. This article offers an analysis of that topic in the historiography of English Protestantism. In the first place, the paper will portray the treatment given by the historiography, considering the criticisms it received at different times. Secondly, it will expose recent advances in the field of
\end{abstract}


history of emotions that could suggest a better approach. It will also highlight the need to observe the polemical dimension within the discourses about Protestant affliction. Finally, it will argue that the study of that affliction requires a special consideration of the context of production, and of the specific vocabulary of the primary sources.

Keywords: Max Weber, Protestantism, affliction, despair, melancholy.

"pues este es el amor a Dios: que guardemos sus mandamien-

tos; y sus mandamientos no son penosos".

1 Juan $5: 3$.

En un sermón publicado póstumamente en 1716, John Sharp, arzobispo de York y consejero personal de la reina Ana, predicó sobre 1 Juan 5:3. ${ }^{1}$ Allí afirmaba:

Algunos tienen unas nociones sobre la religión tan temibles que ciertamente no tienen el coraje para comprometerse sinceramente con ella. Consideran que no está hecha para nadie más que para los melancólicos o los miserables, quienes no están preparados en sus temperamentos para el disfrute de la vida, o quienes por motivo de sus circunstancias infelices están incapacitados para ellos (Sharp, 1716, p. 334). ${ }^{2}$

Para Sharp, la religión verdadera no era melancólica. Por cierto, exigía arrepentimiento y una tristeza genuina por los pecados, pero eso era solo el camino para una felicidad más plena pues, en definitiva, como decía el apóstol, los mandamientos de Dios no eran penosos. ${ }^{3}$ Esta aclaración era recurrente en los escritos de muchos contemporáneos piadosos. Alrededor de la época en que se publicó aquel sermón, Susanna Annesley Wesley se lamentaba en su diario por "cuán injustamente la parte profana del mundo acusa a la religión de melancolía y taciturnidad". Admitía que "puede suceder que las personas religiosas a veces sean obstinadas y taciturnas, pero me atrevo a decir que no es la religión sino la falta de ella la que los hace así" (S. Wesley, 1997, p. 220). ${ }^{4} \mathrm{Su}$ argumento expresaba un ascetismo riguroso:

Lo que sea que disfrutemos de las cosas buenas de esta vida está acompañado de tanta "vanidad y aflicción de espíritu" [Ec. 1, 14; 2, 17] que cualquier persona inteligente percibirá fácilmente que nuestra felicidad incluso en este mundo depende completamente del favor de Dios, el cual no podemos esperar disfrutar sin una práctica constante de la piedad y la virtud (S. Wesley, 1997, p. 220). ${ }^{5}$

1 Este artículo es producto de la investigación para mi tesis doctoral "El mal inglés: melancolía y modernidad en Gran Bretaña, 1660-1750", defendida en la Facultad de Filosofía y Letras de la Universidad de Buenos Aires en julio de 2021. Agradezco a Nicolás Kwiatkowski, Paula Seiguer y los evaluadores anónimos de la revista por sus comentarios a versiones preliminares de este trabajo y a Paula Pico Estrada por su ayuda para acceder a la carta de Martín Lutero citada en la nota 9.

2 "Some have so frightful Notions of Religion, that indeed they have not the Courage in good earnest to engage in it. They look upon it as made for none but either the Melancholy or the Miserable, such as are not framed in their Tempers for the enjoyment of Life, or such as by reason of their unhappy Circumstances are incapacitated for them". A menos que se indique lo contrario, las traducciones son propias.

3 Para la cita del epígrafe se empleó la traducción Reina Valera Antigua. La edición 1960 cambió el texto a "sus mandamientos no son gravosos" y otras versiones enfatizan el sentido de que ellos no son pesados ni difíciles de obedecer. Sin embargo, el texto de la King James Bible dice "his commandments are not grievous", donde grievous tiene ese mismo sentido de "pesado" pero también hace referencia a algo que causa o se caracteriza por una pena o tristeza profundas. En este caso, la traducción elegida permite reflejar este significado que es acorde al sermón de John Sharp.

4 "How unjustly does the profane part of the world charge religion with melancholy and moroseness", "it may sometimes happen that religious persons may now and then be peevish and morose, but I dare say "tis not religion, but their want of it that makes them so". De las 255 entradas de los diarios que se conservan de Susanna Wesley, solo unas pocas tienen fecha. Ésta en particular no tiene pero es de las primeras (la 24, según la numeración de Wallace) y está en un manuscrito (Headingley MS A) escrito entre 1709 y 1718 (S. Wesley, 1997, p. 199).

5 "Whatever we enjoy of the good things of this life are attended with so much 'vanity and vexation of spirit' that any considering person may easily perceive that our happiness even in this world does entirely depend on the favour of 
Susanna es recordada principalmente como la madre y una de las principales guías espirituales de los fundadores del movimiento metodista, John y Charles Wesley. Sin embargo, su religiosidad austera se explica en buena medida por su trayectoria personal y de fe anterior, que la condujo desde el corazón mismo de la comunidad disidente de Londres, donde se había criado, hasta los sectores más conservadores pero no menos ascéticos de la Iglesia de Inglaterra. ${ }^{6}$

La afirmación de Sharp y, especialmente, el caso de Susanna son un buen punto de partida para revisar un problema historiográfico, que es el objeto de este artículo. En los estudios históricos sobre el protestantismo en general, y sobre el inglés en particular, existe una extensa tradición que identifica distintas denominaciones religiosas, en especial el calvinismo, con la producción de un sufrimiento psicológico profundo y patológico. Este topos deriva en buena medida de la obra de Max Weber, que tuvo una influencia notable en la historiografía británica desde muy temprano. Según el sociólogo alemán, que prestó particular atención a los escritos de calvinistas y metodistas ingleses de los siglos XVII y XVIII, la piedad ascética de protestantes como Susanna fue causante de una profunda angustia espiritual. Sin embargo, esta idea no era invención de Weber. Cuando Sharp o Wesley defendían su fe de la acusación de producir melancolía respondían a otras voces contemporáneas que, para deslegitimar las creencias fervorosas, afirmaban cosas similares a las que diría el sociólogo alemán.

Este artículo realiza un análisis historiográfico de ese problema. El objetivo no es ofrecer una respuesta normativa acerca de la relación entre protestantismo y aflicción ni una reevaluación de la tesis de Weber en su conjunto. En cambio, este trabajo se propone, en primer lugar, trazar el derrotero de ese topos en la historiografía sobre el protestantismo inglés y dar cuenta de las críticas que recibió. En segundo término, presenta algunos aportes que se han realizado recientemente desde el campo de la historia de las emociones, que podrían permitir un enfoque superador. En tercera instancia, el artículo argumenta a favor de enfatizar la dimensión polémica de los discursos acerca de la aflicción protestante y sostiene que para estudiarla es preciso observar especialmente el contexto de producción y el vocabulario específico de las fuentes primarias.

\section{La ética protestante y el espíritu melancólico}

En La ética protestante y el espíritu del capitalismo (1904-1905), Max Weber decía del calvinismo: "Con su inhumanidad patética, esta doctrina había de tener como resultado en el ánimo de una generación que la vivió en toda su grandiosa consecuencia el sentimiento de una inaudita soledad interior del individuo" (Weber, 2004 [1905], p. 86). ${ }^{7}$ En el tipo ideal del protestantismo ascético -cuyos efectos creía advertir en

God, which we cannot hope to enjoy without the constant practice of piety and virtue".

6 Susanna era hija del reconocido pastor presbiteriano londinense Samuel Annesley. Por eso, ha sido considerada una figura central en la transmisión de la tradición puritana al metodismo (Newton, 1968, p. 17). No obstante, a los doce años decidió abjurar del inconformismo paterno y se incorporó a la Iglesia de Inglaterra, donde terminó adhiriendo a las ideas religiosas y políticas más conservadoras de la high church y trabó relación con non-jurors como George Hickes. En 1688, se casó con Samuel Wesley, quien había tenido un devenir similar: hijo y nieto de puritanos, se convirtió en sacerdote de la iglesia establecida y habría participado en la defensa del controvertido Henry Sacheverell (Rack, 2004; Torpy, 2009; Tyerman, 1866). Finalmente, la piedad austera de Susanna se reforzó ante una vida familiar difícil, con enfermedades y crisis económicas, en las parroquias rurales de South Ormsby y Epworth en Lincolnshire, adonde siguió a su marido, con quien tuvo diecinueve hijos de los cuales solo nueve llegaron a edad adulta. Existen numerosas biografías de Susanna que han destacado especialmente su vínculo con el metodismo y su papel en la formación espiritual de sus hijos (véanse Kirk, 1865; Clarke, 1886; Harrison, 1938; Harmon, 1968; Newton, 1968; Dallimore, 1993; S. Wesley, 1997; Wallace, 2010).

7 Adapto aquí la traducción de la expresión alemana “Vereinsamung des einzelnen Individuums" (Weber, 2016, pp. 85-86) (en adelante, las citas proceden de la traducción castellana citada en la bibliografía y entre corchetes se 
las naciones capitalistas más avanzadas aún a principios del siglo XX (Weber, 2004 [1905], pp. 15-25 [29-38])-, la presencia de una angustia profunda vinculada con la certitudo salutis constituía un rasgo central.

Weber no fue el único en plantear que el protestantismo, en sus diversas denominaciones, podía ser la causa de un sufrimiento psicológico profundo. ${ }^{8}$ De hecho, afirmaciones similares habían sido frecuentes en las polémicas religiosas desde el siglo XVI. Diversos teólogos habían advertido, en especial, acerca de los efectos dañinos de la especulación acerca de la predestinación. En 1545, el propio Martín Lutero había dicho en una carta del 8 de agosto que "si uno se atormenta constantemente con la providencia, entonces no puede ganar más que angustia" (Luther, 2002, p. 166). ${ }^{9}$ Casi un siglo más tarde, Robert Burton incluyó esa misma preocupación entre las causas de la melancolía religiosa en su Anatomy of Melancholy, donde comentaba que:

Esta curiosidad furiosa, especulación innecesaria, meditación infructuosa acerca de la elección, la reprobación, el libre albedrío, la gracia, tales lugares de las Escrituras concebidos absurdamente, todavía atormentan, y crucifican las almas de demasiados [hombres] y ponen a todo el mundo en desacuerdo (Burton, 2001 [1621], p. 421 [III.4.II.6]). ${ }^{10}$

En cualquier caso, la tesis del sociólogo alemán fue especialmente influyente en la historiografía sobre el protestantismo inglés, por lo que conviene describirla en mayor detalle. ${ }^{11}$

La angustia (Angst) ocupaba un lugar central en el tipo ideal del protestantismo ascético que elaboró Weber (2004 [1905], p. 90 [88]). Su perspectiva estaba fuertemente centrada en el calvinismo pero incorporaba también en su esquema al pietismo y el metodismo. Para el sociólogo, la angustia derivaba fundamentalmente del problema de la certitudo salutis: el tormento continuo acerca de la salvación del alma, compartido por las tres confesiones. ${ }^{12}$ En la doctrina calvinista, la aflicción estaba exacerbada por la distancia inconmensurable entre la divinidad y su criatura, la cual dejaba al individuo en soledad camino a un destino que ignoraba y que debía recorrer sin la ayuda del predicador, de los sacramentos, de la iglesia, ni de Dios que, en última instancia, se había sacrificado solo por los elegidos (Weber, 2004 [1905], pp. 86-87 [8586]). De esta situación surgía un "individualismo desilusionado y pesimista" (Weber, 2004 [1905], pp. 87-88 [86]).

indica el número de páginas de la versión alemana). Como fundamento de esta afirmación, Weber remitía a una frase del crítico literario irlandés Edward Dowden acerca del Pilgrim's Progress de John Bunyan: "The deepest community [with God] is found not in institutions, or corporations, or Churches, but in the secrets of the solitary heart" (Dowden, 1910, p. 234).

8Por ejemplo, Émile Durkheim ya había asociado el individualismo religioso de los protestantes con una mayor tendencia al suicidio (Durkheim,2002 [1897], p. 19). Por su parte, en 1925, Walter Benjamin vio en el luteranismo el germen de la melancolía barroca (Benjamin, 2012 [1925], pp. 179-180). Finalmente, en 1941, Erich Fromm afirmaría que la angustia, la soledad y la impotencia eran algunas de las consecuencias necesarias de la nueva libertad inaugurada por la Reforma (Fromm, 1973 [1941], Capítulo 3).

9 “Denn wann man ewig sich mit der Vorsehung martert, So gewinnet man doch nichts daran, dann Angst”.

10 "this furious curiosity, needless speculation, fruitless meditation about election, reprobation, free will, grace, such places of Scripture preposterously conceaved, torment still, and crucify the souls of too many and set all the world together by the ears".

11 Sobre la recepción del libro de Weber, véase Gil Villegas (2013).

12 En el calvinismo la angustia de la certitudo salutis derivaba directamente de la doctrina de la doble predestinación (Weber, 2004 [1905], p. 96 [92-93]). En el pietismo, “las mismas consecuencias de orden psicológico" resultaban de la conformación de una aristocracia de los regenerados, bendecidos con el éxito profesional, en quienes la gracia irrumpía en momentos particulares luego de una "lucha expiatoria” (Bußkampf) (Weber, 2004 [1905], p. 126 [119]). Por último, el énfasis sentimental del metodismo wesleyano, especialmente en su versión americana, habría exacerbado la lucha expiatoria del pietismo (en el "banco de los penitentes", que Weber Ilamaba Angstbank) y, como los calvinistas, habría postulado la “'metodización' sistemática de la conducta como medio de alcanzar la certitudo salutis” (Weber, 2004 [1905], pp. 133-134 [126-127]). 
Weber desarrolló las consecuencias de esta doctrina en la edición revisada de su obra, publicada póstumamente en $1920 .{ }^{13}$ Desde su punto de vista, el catolicismo había preservado el papel de la magia como medio de salvación más que el judaísmo o el puritanismo. Los sacerdotes católicos eran, pues, magos que administraban la gracia sacramental de la Iglesia. Eso permitía compensar la insuficiencia propia de los fieles y liberarlos de una "tensión enorme" (ungeheuren Spannung). Esta posibilidad quedaba excluida para los puritanos, quienes rechazaban esas mediaciones como ineficaces y supersticiosas. Según el sociólogo, el abandono absoluto de la posibilidad de salvación eclesiástico-sacramental supuso la conclusión (Abschluß) del extenso proceso de desencantamiento del mundo (Entzauberung der Welt) (Weber, 2004 [1905], pp. $87 \mathrm{y}$ 105 [200 y 208]). ${ }^{14}$ En otras palabras, la angustia inaudita de los calvinistas era efecto de una doctrina racionalizadora que execraba la magia y anticipaba las condiciones de existencia en un mundo moderno y secular donde la vida ya no tendría un sentido intrínseco. ${ }^{15}$

Sin embargo, la angustia no era un punto de llegada. A partir de la lectura de tratados de teología práctica como el Christian Directory (1673) de Richard Baxter, Weber concluía que la religiosidad ascética invitaba a rechazar las dudas sobre la elección como una tentación del diablo y fomentaba el trabajo secular como modo de obtener seguridad acerca del estado de gracia. Las obras, aunque no constituían un medio para la salvación, eran indispensables como signo de elección de aquellos llamados a ser instrumentos de la divinidad. La bienaventuranza no se verificaba en actos individuales sino allí donde había un sistema de conducta que servía para aumentar la gloria de Dios. La ética protestante, por lo tanto, era un medio para desprenderse de la angustia y para producir santos seguros de sí mismos (Weber, 2004 [1905], pp. 100-102 [96-97 y 207]).

La obra de Weber ejerció una influencia notable en historiadores británicos como Richard H. Tawney, Daniel P. Walker, Christopher Hill y Keith Thomas, entre muchos otros. Ellos retomaron en ocasiones la imagen del puritanismo caracterizado por una aflicción derivada de sus principios doctrinales, así como también la premisa de que el rechazo de las mediaciones salvíficas propició el desencantamiento del mundo y dejó a los fieles a merced de una angustia que debía ser mitigada por otros medios. Así, en una obra clásica de 1926 que dialogaba explícitamente con la de Weber, Tawney describió al puritano como "abrumado por una sensación de su 'Fin Último'" pero vedado de detenerse en la contemplación y obligado a "glorificar [a Dios] con su trabajo en un mundo entregado a las fuerzas de la oscuridad" (Tawney, 1937, p. 200). Por su parte, Walker imaginó que "la abolición del purgatorio [debía haber] producido para muchos protestantes una situación dolorosa" (Walker, 1964, p. 59). Christopher Hill retomó estos aportes, afirmó que el protestantismo se caracterizaba

13 Sobre algunas experiencias personales significativas de Weber entre ambas ediciones, véase Bartra (2018 [2004]). 14 Este concepto ha sido objeto de numerosas apropiaciones, resignificaciones y críticas que se volcaron en una extensa bibliografía (sin ánimo de ofrecer una lista exhaustiva, véanse Scribner, 1993; Asprem, 2014; Parish, 2015; Jason, 2017). Algunos autores relativizaron la relevancia del protestantismo en este proceso. Por ejemplo, para Marcel Gauchet "el "protestantismo ascético", tan estimado por Max Weber, no constituye más que una franja pionera y la fracción emergida de un inmenso movimiento subterráneo" (Gauchet, 2005, p. 115). Por su parte, Slavoj Žižek sostuvo que el reconocimiento angustiante de la inexistencia de una garantía externa para el éxito era la vía particular del protestantismo al ateísmo, comparable con otras presentes en el judaísmo, el catolicismo y el islam. Aunque, en última instancia, su argumento más controvertido es que el cristianismo en su conjunto, más que cualquier otra religión, contiene el germen del ateísmo en las palabras de Cristo en la cruz: "Dios mío, ¿por qué me has desamparado?" (Mateo 27, 46; Marcos 15, 34) (Žižek, 2009, pp. 48-49).

15 En La ciencia como vocación de 1918, Weber había definido el desencantamiento del mundo como un proceso de racionalización e intelectualización vinculado con el mayor dominio sobre la naturaleza que, sin embargo, no implicaba un conocimiento mayor de las condiciones de vida. Para eso partía de León Tolstoy quien decía que, para el hombre civilizado (Kulturmensch), la muerte no tiene sentido pues su vida está inserta en el progreso y lo infinito y, por lo tanto, no tiene ningún significado intrínseco. En ese proceso continuo de producción y adquisición de conocimiento, donde todas las nuevas ideas se vuelven finalmente provisionales, el hombre puede llegar a estar cansado de la vida (lebensmüde) en vez de satisfecho con ella (lebensgesättigt) (Weber, 1992, 2004). 
por la internalización del pecado, cuyos terrores se inculcaban en la conciencia y, luego, resaltó una diferencia de clase que atravesaba la ética protestante al destacar que, especialmente para los desfavorecidos por la divinidad, "la desesperación y las ideas de suicidio [...] pueden ser subproductos del calvinismo" (Hill, 1975, pp. 152, 155, 170 y 328 ). Keith Thomas llevó la categoría weberiana del desencantamiento del mundo a un estudio monumental de la "decadencia de la magia" en la Inglaterra temprano moderna. Allí están ampliamente desarrolladas las críticas de los protestantes a las mediaciones sacramentales y la idea -tomada de Bronislaw Malinowski- de la magia como un modo de aliviar la ansiedad. También en la sección dedicada a la demonología afirmaba que "el protestantismo era una respuesta a una profunda convicción del pecado humano, una sensación de impotencia ante el mal". Sin embargo, no he podido encontrar referencias explícitas a que el protestantismo generara un aumento extraordinario de la angustia (Thomas, 1991 [1971], pp. 315-316, 560-561, 774-775, 800).

En estos textos, la aflicción protestante desempeñaba un papel secundario, no tenía el mismo potencial explicativo que le daba Weber y quedaba relativizada en explicaciones que enfatizaban la seguridad y energía que la certidumbre de la salvación proveía a los calvinistas. En efecto, en un artículo donde abordaba las tesis de Weber y Tawney, Christopher Hill escribió que lo que el protestantismo le había dado al "hombre escrupuloso del siglo XVI" fue

una calma interior y una confianza en sí mismo, quizás intermitentes, pero basadas firmemente en momentos de júbilo los cuales, una vez experimentados, distinguían a un hombre a sus propios ojos frente a su prójimo. (De allí la importancia de la doctrina de que los elegidos no podían nunca caer completamente de la gracia) (Hill, 1961, p. 24).

Más adelante decía que "el sentimiento del pecado permaneció, se hizo incluso más sobrecogedor porque se debía luchar solo contra él. Pero el sentimiento de pecado era también ahora un sentimiento de potencial libertad" (Hill, 1961, p. 38). El historiador inglés también destacó el efecto movilizador de la doctrina de la predestinación en su biografía de Oliver Cromwell (Hill, 1990 [1970], Capítulo IX) y, en su estudio sobre John Bunyan, relativizó el problema al plantear que "la crisis económica de principios del siglo XVII puede haber cooperado con el calvinismo para inducir un sentido de desesperación: sería inútil especular cuál fue la causa más significativa" (Hill, 2016 [1988], p. 185).

En otros estudios, en cambio, la preocupación weberiana por la aflicción fue central. Tal es el caso de The Revolution of the Saints (1965) de Michael Walzer, quien presentó la superación terrenal de la angustia como un rasgo central de la ideología calvinista que, además de estimular vocaciones seculares, daba lugar a una acción política sistemática y militante (Walzer, 1963; 1965, pp. 13, 24-25). Para Calvino, decía el autor, "el temor y la angustia" formaban parte de las "experiencias clave del hombre caído" y la cura había que buscarla no en la reconciliación sino en la obediencia (Walzer, 1965, pp. 27-28). A partir de un argumento ya planteado por William Haller, Walzer afirmaba que si bien "probablemente no es cierto que el calvinismo causara angustia", ofrecía una explicación teológica de los peligros en una época de cambios desconcertantes: "la santidad ofrecía una salida de la angustia" (Haller, 1938, p. 27; Walzer, 1965, p. 308). Siguiendo a Weber planteaba, entonces, que la ética protestante permitió fortalecer el carácter de los santos para que pudieran desempeñar su "papel dramático" en la "crisis de la modernización" (Walzer, 1965, pp. 18-19).

A principios de la década de 1990, el crítico literario John Stachniewski propuso una tesis que en algunos aspectos circunscribía y en otros profundizaba y radicalizaba la asociación del calvinismo con la angustia. El límite consistía en que se concentraba 
exclusivamente en el puritanismo inglés del siglo anterior a la Restauración. Sin embargo, buscaba trascender lo que consideraba una perspectiva demasiado optimista (up-beat) derivada de Weber y afirmaba que el calvinismo había producido una cultura opresiva y autoritaria caracterizada por una "imaginación persecutoria", que había sido determinante en la construcción de subjetividades y había multiplicado la experiencia de la desesperación religiosa (Stachniewski, 1991). ${ }^{16}$ Esta tesis era completamente contraria a la de Weber, para quien el calvinismo era una doctrina fundamentalmente anti-autoritaria (Weber, 2004 [1905], pp. 89-90, n. 113 [202]), y a las de Haller y Walzer, quienes habían visto en el puritanismo una ideología revolucionaria, modernizadora y reconfortante.

Recientemente, la teoría de Stachniewski fue refrendada por James Simpson en un estudio sobre las "raíces intolerantes del liberalismo". Allí afirmó que "las formas más dinámicas y difundidas de la modernidad temprana noreuropea comenzaron con la desesperación" y realizó una descripción deliberadamente pesimista de lo que llamó la "producción calvinista de depresión patrocinada por el Estado" (Simpson, 2019, pp. 57 y 72). Las interpretaciones de Stachniewski y Simpson fueron criticadas duramente por Christopher Hill y Keith Thomas respectivamente, quienes sostuvieron que aquellas enfatizaban una caricatura del puritanismo a partir de un uso selectivo de fuentes, en su mayoría, literarias (Hill, 1993; Thomas, 2020).

Por su parte, en 1994, el sociólogo Julius Rubin recuperó la tesis weberiana para integrarla en una "etnopsiquiatría" de la melancolía religiosa. Según el autor, el problema de Weber era que se concentraba en el creyente que heroicamente lograba superar el dilema existencial protestante y, de ese modo, perdía de vista hasta qué punto el ascetismo generaba una propensión a esa enfermedad (Rubin, 1994, p. 18). Desde la perspectiva de Rubin, la melancolía religiosa era una experiencia típica entre los grupos protestantes desde el siglo XVII hasta el XIX. Por eso, se refería a ella como un "síndrome cultural" (culture-bound syndrome) "vinculado inextricablemente con la conversión religiosa y el significado de la relación del creyente con Dios" (Rubin, 1994, p. 10).

La noción de "síndrome cultural" es una categoría que puede ser útil para referirse a trastornos que surgen en universos simbólicos particulares y, en este caso, para dar cuenta de la relación específica entre protestantismo y aflicción. ${ }^{17}$ Por ejemplo, Matthew Bell sostuvo que la melancolía es un síndrome cultural occidental (Bell, 2014, Capítulo 4). No obstante, eso es distinto a pensar en una cultura que produce sistemáticamente una enfermedad como una experiencia típica.

Por otro lado, Rubin era plenamente consciente de que "la categoría diagnóstica de melancolía religiosa proveía un instrumento cargado políticamente para desacreditar a la heterodoxia" (Rubin, 1994, p. 6). Sin embargo, no dudaba en realizar su propio diagnóstico al identificar los síntomas de esa "forma cultural e históricamente específica de trastorno depresivo" incluso en fuentes que negaban explícitamente que las experiencias religiosas que describían fueran producto de la melancolía (Rubin, 1994, p. 3). Esto resulta problemático pues implica atribuir valor analítico a una categoría nativa concebida con fines polémicos. ${ }^{18}$ En efecto, la noción de "melancolía religiosa"

16 Otro autor que discute esta idea de Weber es Zafirovski (2007).

17 Es preciso destacar los orígenes etnocéntricos de esta categoría que fue empleada, a menudo, para designar enfermedades que se observaban solo en sociedades no-occidentales, mientras que los síndromes que se registraban en Europa o Estados Unidos tendían a considerarse universales. Sin embargo, en la actualidad también se utiliza para referirse a patologías occidentales no generalizables. Tal es el caso del uso que hace Rubin (sobre esta categoría, véanse Simons \& Hughes, 2012 [1985]; 1993; Kleinman, 1988; 2020).

18 Como ha señalado Ginzburg, al perder de vista la distinción entre las categorías nativas y las de los historiadores, entre los niveles de análisis emic y etic, se corre el riesgo de caer en la empatía y la ventriloquía de las personas del 
fue acuñada por Burton en la Anatomy of Melancholy para caracterizar los desvíos en el justo amor a Dios (por exceso o por defecto) de católicos, puritanos y ateos (Burton,2001 [1621], pt. III, sect. IV; Gowland, 2006a, Capítulo 3).

Los ejemplos anteriores no agotan las referencias que la historiografía y las ciencias sociales preservan sobre la relación entre protestantismo y aflicción patológica. Sin embargo, son suficientes para mostrar la relevancia del problema y su asociación con las explicaciones acerca del origen de la modernidad.

\section{El malestar en la cultura protestante}

No se puede negar que la angustia y otras formas de sufrimiento psicológico tienen una presencia significativa en las elaboraciones doctrinales y en la cultura protestantes. Esto se verifica, por ejemplo, como había planteado Weber, en la concepción de Calvino de una humanidad totalmente depravada, pero también en el concepto luterano de Anfechtung (Scaer, 1983; cfr. Stachniewski, 1991, p. 18), en las fases depresivas de las autobiografías espirituales (Hodgkin, 2007) o en los peligros que encontraba Cristiano en The Pilgrim's Progress: ese peregrinaje alegórico que se convirtió en ejemplar para varias generaciones de protestantes, donde el protagonista se encontraba con el Pantano del Desaliento o el Castillo de las Dudas donde moraba el gigante Desesperación (Bunyan, 1996 [1678], pp. 13, 92; Dunan-Page, 2006; Stachniewski, 1991, Capítulo 4). Sin embargo, plantear esto como una prerrogativa exclusiva del protestantismo o como un resultado directo de sus doctrinas tiene una serie de problemas que la historiografía ya ha señalado. ${ }^{19}$

En primer lugar, se ha discutido si la aflicción desempeñaba efectivamente un papel tan central en la experiencia de los protestantes. En 1954, el escritor C. S. Lewis afirmó que las doctrinas del puritanismo no eran "de terror sino de gozo y esperanza" y seleccionó convenientemente una serie de citas que le permitieron afirmar que "fueran lo que fueran, [los primeros protestantes] no eran amargados, tristes ni severos, ni sus enemigos los acusaban de algo así" (Lewis, 1954, pp. 33-34). ${ }^{20}$ Varios autores insistieron en que la consecuencia de la doctrina de la doble predestinación calvinista no era la angustia sino, por el contrario, el sosiego ${ }^{21}$ Más recientemente, Patrick Collinson señaló la imposibilidad de saber si ese tipo de aflicción estaba tan difundido como para ser característico de la experiencia calvinista como consideraba Weber, pues "la ausencia de angustia, como la conformidad convencional, tiene poca o ninguna historia" (Collinson, 2003, p. 135). Ese contraste entre la "imagen intelectualizada" que procede del estudio de la ideología y la experiencia real aparecía también en la obra de Edward P. Thompson. Por eso, aunque caracterizaba al metodismo de fines del siglo XVIII como "una forma ritualizada de masturbación mental" y de "terrorismo religioso" (siguiendo a Lecky, 1921 [1892], p. 77), el historiador era consciente de que, en la práctica, el movimiento creaba comunidad y el dogma podía ser suavizado, humanizado y adaptado de acuerdo con las necesidades, valores y relaciones sociales concretas (Thompson, 1966, Capítulo 11, esp. pp. 368-369 y 379-380).

pasado (2012, p. 109). En este caso, se trataría de una empatía con el discurso antipuritano. Al respecto, véanse también los comentarios de MacDonald más abajo.

19 Además de las críticas que se plantean a continuación, véanse los comentarios de Bell (2014, pp. 124-125), Gowland (2006b, pp. 105-106) y Schmidt (2007, pp. 49-64).

20 Stachniewski, de hecho, plantea su postura por oposición a la de Lewis (Stachniewski, 1991, pp. 1-5).

21 Además de los textos de Hill, Haller y Walzer ya citados, véase Crouzet (2001, pp. 157-162). Nathan Johnstone, aunque está de acuerdo con Stachniewski en varios puntos, considera que la idea de que el protestantismo fomentara un miedo obsesivo a Satán y dejara a sus fieles desprotegidos contra el demonio es engañosa (Johnstone, 2006, pp. 84-85). 
En segundo lugar, con respecto a la angustia de los puritanos ingleses en particular, se ha planteado que las causas de su aumento quizás deberían buscarse tanto o más en la crisis económica y social del siglo XVII que en la doctrina calvinista (Hill, 1993, p. 97, 2016 [1988], p. 185). Vale la pena recordar que Hugh Trevor-Roper, en su célebre debate con Eric Hobsbawm, había incluido entre los indicadores de que la crisis del siglo XVII no había sido solamente política y económica "la difusa sensación de pesimismo [gloom] que percibimos constantemente en esos años" (Trevor-Roper, 1959, p. 31).

Por último, la asociación exclusiva de la angustia con el protestantismo implica ignorar hasta qué punto el énfasis de la aflicción como medio para alcanzar el arrepentimiento y probar la fe formaba parte de una tradición común al cristianismo en su conjunto. Así, en el Antiguo Testamento es posible encontrar numerosas referencias al carácter necesario o benéfico de la aflicción. Por ejemplo, las descripciones vívidas del sufrimiento del salmista cuando Jehová escondía su rostro de él (Sal 88:14) y las afirmaciones recurrentes del poder de Dios para liberar al hombre de la angustia (Sal 43; Sal 50:15; Sal 59:16; Sal 69; Sal 107:28). A esos pasajes se podría sumar la sentencia del Predicador según la cual "Mejor es el pesar que la risa, porque con la tristeza del rostro se enmienda el corazón" (Ec 7:3) o figuras como la de Job o el "varón de dolores" descrito por Isaías (Is 53:1-12), que mostraban que las penas templan a los justos y anticipan sus bendiciones futuras. Esta noción se repetía en el Nuevo Testamento cuando Pedro se refería a la aflicción como una prueba de fuego para la fe (1 P1:7) y en la segunda carta a los Corintios, donde Pablo decía que "la tristeza que es según Dios produce arrepentimiento para la salvación" (2 Co 7:10).

Esto solo por limitarse a ejemplos del texto bíblico. Desde un punto de vista diferente pero que reforzaba las similitudes entre confesiones cristianas, Jean Delumeau argumentó que el énfasis en la culpa, el miedo y la angustia respondía a una tendencia general de la cultura occidental entre los siglos XIII y XVIII, vinculada con un proceso de introspección e interiorización del pecado sin precedentes que dio lugar a una nueva conciencia moral (Delumeau, 1991; 2006; 2017).

Estas apreciaciones obligan a relativizar la centralidad del protestantismo en relación con una angustia que, tanto desde la perspectiva weberiana como de las de Stachniewski y Simpson, estaba asociada con un proceso de racionalización y modernización al que las sociedades católicas habrían llegado de manera tardía y periférica. Sin embargo, esto no impide pensar que los protestantes, en sus diversas denominaciones y con sus particularidades, puedan haber tenido una concepción y una experiencia particular de la angustia y la melancolía. Un estudio de esa naturaleza es valioso pero requiere tomar algunas precauciones metodológicas. Sobre ello versa el resto de este artículo.

\section{De la patología a las emociones}

A partir del recorrido historiográfico anterior, es posible establecer una distinción entre tres tipos de postulados acerca de la relación entre protestantismo y aflicción que no deberían confundirse. Por un lado, en la obra de Weber y algunos de sus seguidores, las doctrinas protestantes aparecen como causantes de una angustia extraordinaria, mayor que las de otras confesiones religiosas y, eventualmente, como proveedoras de una forma igual de excepcional para librarse de aquella. Por otro lado, Stachniewski, Rubin y Simpson plantearon un argumento más radical: que la cultura calvinista generaba sistemáticamente, como una experiencia típica, un sufrimiento patológico. Finalmente, una tercera alternativa es afirmar que el protestantismo, en sus 
diversas variantes confesionales, establecía sentidos, valores y normas de expresión para emociones como la angustia, la tristeza o el miedo. Esto supondría que aquél producía una experiencia afectiva particular, pero no que tenía el monopolio de la angustia ni que era el vector de una enfermedad mental.

Esta tercera perspectiva ha sido explorada en años recientes desde el punto de vista de la historia de las emociones. La reivindicación de la historicidad de las emociones y de su permeabilidad cultural ha permitido afirmar que aquellas no son únicas e iguales en todos los tiempos y espacios. ${ }^{22}$ Ello llevó a pensar en formas de entender los modos en que distintos grupos sociales establecieron normas, valores, sentidos y conceptos diferentes para dar cuenta de su vida afectiva. Así, por ejemplo, en una obra señera, William Reddy postuló la existencia en distintas sociedades de regímenes emocionales: un conjunto de normas, rituales, prácticas y enunciados emotivos (o emotives) que constituyen el sustento de un régimen político determinado (Reddy, 2001, pp. 122-130). Por su parte, Barbara Rosenwein señaló los límites de esa categoría para estudiar sociedades premodernas por su vínculo estrecho con el concepto de Estado y propuso, en cambio, hablar de comunidades emocionales: un agrupamiento de una escala inferior y que permite concebir la coexistencia en tiempo y espacio de comunidades diferentes (Rosenwein, 2006, 2016).

Estos estudios permitieron concebir, por un lado, la existencia de emociones específicamente religiosas (Cummins \& Stille, 2021) y, por otro, que algunas comunidades - protestantes, puritanas, etcétera- pudieran tener emocionalidades particulares, distintas de otras. Por cierto, esto deja abierto el problema que se le criticó a Rosenwein de cómo establecer o identificar las fronteras de una comunidad emocional, una categoría que tiende a ser rígida e imprecisa (Bjerg, 2019, p. 10). Sin embargo, la imprecisión puede ofrecer también una flexibilidad provechosa al momento de indagar en el terreno complejo de la multiplicidad de denominaciones protestantes que, muchas veces, tienen más en común de lo que las enfrenta.

Recientemente, Monique Scheer sostuvo que uno de los puntos de conflicto pero también de similitud entre las diversas confesiones protestantes era la eliminación de mediaciones entre el alma individual y la divinidad, que generaba la paradoja de cómo establecer una comunicación sin medio. Como consecuencia, según la autora, los protestantes -a diferencia del estereotipo corriente que los muestra como portadores de una racionalidad impasible- eran los cristianos más preocupados por las emociones. Esto se debía a que ellas constituían la mediación de su creencia, al proveer un tipo de "materialidad" (incluso cuando se la interpretara como inmaterial) que "sostiene y ratifica la concepción de algo como real y verdadero" (Scheer, 2020, p. 5).

Otro autor que enfatizó los aspectos en común en su estudio de lo que implicaba ser protestante en Gran Bretaña en tiempos de la Reforma es Alec Ryrie. Interesado especialmente por la devoción y la experiencia vivida, argumentó que el protestantismo de la modernidad temprana era una "cultura religiosa amplia" (broad-based religious culture), en la cual las divisiones entre puritanos y conformistas - que tan relevantes han sido para los historiadores- tienden a disolverse (Ryrie, 2013, p. 6). Los trabajos de este autor forman parte de los estudios recientes sobre las emociones del protestantismo británico que se han concentrado especialmente en aquella cultura religiosa que se desarrolló en las islas entre la Reforma y 1640 y en el puritanismo a ambas márgenes del Atlántico (Ryrie E Schwanda, 2016; Rosenwein, 2018; Méndez, 2021).

22 Para una introducción a este campo historiográfico, véanse Oatley (2004), Plamper (2012), Boddice (2018), Bjerg (2019). Con respecto a la interacción entre cultura y afectos, es preciso señalar que la historiografía estuvo a la zaga de investigaciones fundantes de la antropología de las emociones como Rosaldo (1980), Lutz \& Abu-Lughod (1990), Lutz (1998). 
A los efectos del problema planteado en este artículo, es relevante destacar que Ryrie se opuso tajantemente a la idea de Stachniewski del protestantismo inglés como una cultura de la desesperación e incluso afirmó que "fue Weber, sobre todo, quien creó el mito del calvinista desesperado, y nosotros no deberíamos necesariamente creer en él". Además, advirtió respecto del peligro de dejarse engañar por la abundancia de fuentes sobre el tema: "la ansiedad acerca de la salvación produjo una gran cantidad de papel, pero eso no nos habla de la escala del problema" pues -de modo similar a lo que había dicho Collinson- quienes "no estaban perturbados o estaban efectivamente reconfortados por la doctrina de la predestinación, no necesitaban que se escribieran cartas o tratados para calmar sus conciencias" (Ryrie, 2013, p. 29). Por cierto, la felicidad y la alegría también eran deberes cristianos y, aunque los protestantes de distintas denominaciones se apuraran a aclarar que no se trataba de un gozo mundano, eso no implicaba que fuera un rasgo infrecuente ni puramente teórico de sus vidas (Ryrie, 2013, pp. 77-80). El autor también invitó a distinguir entre enunciados que describían una experiencia de fe concreta y aquellos otros que eran declaraciones teológicas acerca de cómo aquella podía o debía ser (Ryrie, 2013, p. 36). Por otro lado, con respecto a la experiencia puritana de la desesperación en particular, Ryrie sostuvo que lo distintivo era su autoconciencia y no su naturaleza: "los puritanos del siglo XVII describieron tales experiencias con mayor detalle que otros protestantes, pero no tenían un contrato exclusivo con ellas" (Ryrie, 2013, p. 39).

\section{Texto, contexto y vocabulario}

Las citas de John Sharp y Susanna Wesley al comienzo de este artículo son testimonio de que, por entonces, el efecto -patológico o no- de la religión sobre las emociones era objeto de disputa. Por eso, al momento de valorar la experiencia afectiva de los hombres y mujeres del pasado, es preciso también tomar precauciones en la interpretación de las fuentes. Juzgar adecuadamente las expresiones acerca de la aflicción de los protestantes requiere, antes de aceptarlas como descripciones confiables de la realidad, considerar las características particulares de cada enunciado, su contexto de producción y las especificidades del vocabulario empleado.

Los historiadores de las emociones no ignoran estos aspectos, aunque a menudo pueden quedar relegados o implícitos en aquellos enfoques que se centran en reconstruir la experiencia vivida de los sujetos. Una reflexión más sistemática al respecto puede servir para poner de relieve la relevancia de la dimensión polémica en la construcción de sentidos acerca de las emociones.

En primer lugar, en cuanto al tipo de enunciado, es preciso reconocer que no eran iguales el tono, la intencionalidad ni el destinatario de un manual de devoción que el de una autobiografía espiritual, un sermón, una consolación de la melancolía o un texto de polémica teológica, y no pueden leerse del mismo modo. Así, mientras que en el tipo de obras literarias y autobiográficas que estudió Stachniewski la angustia por el destino trascendente de las almas estaba en primer plano, al observar otro tipo de fuentes como testamentos e inscripciones funerarias se obtiene una imagen completamente diferente: la de una población que, a pesar de las enseñanzas más severas de los púlpitos, era optimista con respecto a su salvación (Thomas, 2009, pp. 232-233). Además, Ryrie señaló que los diarios y las autobiografías, a pesar de que son algunas de las mejores evidencias disponibles sobre la experiencia interior de las personas, por su naturaleza prestan mayor atención a los cambios y las variaciones que a los estados constantes (Ryrie, 2013, p. 82). En un sentido similar, en otra parte apuntó que la función de los diarios espirituales era llevar un registro de los pecados, pero eso "no quiere decir que estés obsesionado con el pecado, del mismo 
modo que tener un libro de contabilidad no significa que estés obsesionado con el dinero" (Ryrie, 2013, p. 29). En otras palabras, aquella atención era evidencia de la relevancia que tenían el pecado y el arrepentimiento en la cultura protestante, pero no necesariamente de una fijación patológica.

Por otro lado, este tipo de textos tenía como objetivo presentar una imagen piadosa de sus protagonistas y eso implicaba narrar sus vidas de acuerdo con ciertas experiencias arquetípicas (Hodgkin, 2007, p. 25). Jeremy Schmidt señaló que para los protestantes ingleses del siglo XVII -privados de afirmar la gracia a través de las obras como los católicos-, el miedo y la tristeza que derivaban del reconocimiento de la depravación eran "tanto la sustancia de la piedad como el medio para la certeza de la salvación". Siguiendo a Peter Kaufman, señaló que "la desesperación religiosa de fines del siglo XVI y principios del XVII puede ser entendida en gran medida como un efecto performativo de la oración y la meditación" (Schmidt, 2007, p. 54; cf. Kaufman, 1996, p. 18). Esta afirmación puede interpretarse de dos modos que no suponen realidades mutuamente excluyentes. Por un lado, es posible que, para algunos, la oración y la meditación tuvieran como efecto la desesperación. Por otro lado, presentarse como afectado por ese estado emocional podía ser una forma de mostrar públicamente la propia santidad. En ese sentido, algunos autores se han referido a la capacidad de los puritanos de dar sentido a los momentos de desesperación en sus autobiografías como un modo de self-fashioning (MacDonald, 1992, pp. 56-58; Sullivan, 2016, pp. 192-197).

Estas observaciones conducen a la constatación de que para poder interpretar adecuadamente las fuentes, no basta con comprender el significado de los enunciados en sí mismos, sino que es preciso dar cuenta de su dimensión pragmática: de aquello que sus autores hacen al ponerlos en circulación. Por eso, resulta fundamental atender al contexto de producción de esos enunciados (Skinner, 2002). ${ }^{23}$

En relación con esto, se debe recordar que, aunque la mayor parte de la historiografía posterior tendió a enfocarse en el problema de la angustia en la cultura religiosa inglesa entre la Reforma y 1640, el estudio de Weber incluía también al pietismo y al metodismo del siglo XVIII. Además, para referirse al puritanismo, el sociólogo recurría a menudo al testimonio de Richard Baxter, el prominente teólogo de la generación que vivió las guerras civiles y la Restauración, quien tenía algunas ideas bastante diferentes a las de sus antecesores. ${ }^{24}$

Es relevante revisar brevemente el contexto específico de la segunda mitad del siglo XVII y el XVIII pues allí se gestaron sentidos acerca de la afectividad protestante que incidieron en la experiencia de movimientos religiosos nuevos como el metodismo, pero también en la representación histórica de los puritanos de la generación anterior. Así, por ejemplo, en 1712, Joseph Addison escribía en The Spectator ( $\left.\mathrm{N}^{\circ} 494\right)$ :

Hace alrededor de una generación era la moda en Inglaterra que quien quisiera parecer religioso se echara toda la santidad posible en la cara y, en particular, se abstuviera de cualquier apariencia de alegría y buen humor, las cuales eran vistas como marcas de una mente carnal. El santo tenía un semblante triste y estaba generalmente carcomido por el spleen y la melancolía (Addison \& Steele, 1806, p. 112). ${ }^{25}$

23 Desde un punto de vista diferente, a partir de los casos de Richard Baxter y John Bunyan, David Walker señaló la relevancia del contexto político y religioso no con respecto a la producción de sentido, sino por su incidencia en la experiencia emocional (Walker, 2016).

24 La bibliografía sobre Baxter es muy vasta. Véanse especialmente Lim (2004) y Cooper (2011). Sobre sus ideas sobre la melancolía, véase Schmidt (2007, Capítulo 5) y D. Walker (2016).

25 "About an age ago it was the fashion in England, for every one that would be thought religious, to throw as much sanctity as possible into his face, and in particular to abstain from all appearances of mirth and pleasantry, which were looked upon as the marks of a carnal mind. The saint was of a sorrowful countenance, and generally eaten up 
Desde mediados del siglo XVII, la asociación de ciertas creencias y prácticas religiosas con la melancolía era un componente medular del discurso de reprobación y ridiculización del entusiasmo. Éste era un término despectivo para referirse a todos aquellos que declaraban estar inspirados por el Espíritu Santo y reivindicaban la superioridad de la revelación personal sobre la del texto bíblico (Knox, 1950; Heyd, 1981, 1995). No se trataba de una polémica meramente teológica, sino que a partir de la Restauración se convirtió en un concepto clave del lenguaje político que sería fundamental para articular un consenso amplio en torno de la búsqueda de una paz y un orden duraderos (Pocock, 1997). Además, el término amplió sus incumbencias para extenderse a las polémicas sobre la filosofía natural y sobre el lenguaje, hasta convertirse en una de las principales formas de difamación en el siglo XVIII (Williamson, 1933; Shapin ESchaffer, 1985; Laborie, 2015).

Es en ese contexto que debe entenderse la preocupación de personas piadosas como el arzobispo Sharp o Susanna Wesley por defender a su fe del cargo de causar melancolía. El mismo afán se advierte en un libro que ha sido descripto como el que "más influenció el pensamiento religioso inglés del siglo XVIII" (Starkie, 2002, p. 452): A Serious Call to a Devout and Holy Life (1729) de William Law. Allí, el autor dedicaba el capítulo XI a refutar la idea de que una vida devota consagrada a Dios fuera "aburrida, inquieta y melancólica" ${ }^{26}$ (Law, 1729, p. 163). Estos textos buscaban establecer un límite entre la experiencia de fe auténtica y el entusiasmo.

Al mismo tiempo, como se advierte en la cita de Susanna al principio de este artículo, también pretendían diferenciarse de quienes buscaban la felicidad en los placeres mundanos. Para Law, la dicha verdadera no consistía en tener la libertad para ceder a las pasiones y los apetitos, sino en la capacidad de controlarlas. Por lo tanto, "la felicidad real solo se alcanzará con mayores grados de piedad, mayores abstinencias de nuestras pasiones y las más estrictas reglas de la religión" (Law, 1729, p. 165). ${ }^{27}$ Entre ambos extremos, delineaban los contornos de una aflicción espiritual saludable y piadosa.

La definición de los límites y las formas de un sufrimiento legítimo se daba también en el uso del vocabulario. A menudo, los pastores se esforzaban por establecer precisiones entre conceptos que podían resultar confusos para sus feligreses. En efecto, para aquellos no era lo mismo hablar de angustia, tristeza, aflicción, melancolía, pesadumbre o desesperación. En la elección de la terminología también es posible apreciar el intento de distinguir entre estados de ánimo normales y patológicos, y de trazar los límites de una experiencia de fe legítima.

El estudio de las palabras y los conceptos es un foco de interés habitual en la historia de las emociones (Dixon, 2012; Frevert et al., 2014; Pernau \& Rajamani, 2016). Sin embargo, las especificidades y los usos polémicos del vocabulario de la aflicción en el protestantismo inglés es un ámbito que requiere aún un análisis en profundidad. Un primer paso en esa dirección lo dio Michael MacDonald, quien abordó el concepto de desesperación o desesperanza (despair) a partir de la recepción de la historia del apóstata italiano Francesco Spiera en Inglaterra. Según el autor, para los puritanos y los metodistas, aquella era una emoción particular, diferente de la desperatio medieval pero también de la melancolía. Por lo tanto, afirmaba que aplicar esta última etiqueta a la desesperación implicaba reproducir el discurso ortodoxo que buscaba desacreditar la autenticidad de la experiencia de los evangélicos al asimilarla a una enfermedad (MacDonald, 1992, pp. 59-60).

with spleen and melancholy".

26 "Some people will perhaps object, that all these rules of holy living unto God [...] shall render our lives dull, uneasy, and melancholy".

27 "real happiness is only to be had from the greatest degrees of piety, the greatest denials of our passions, and the strictest rules of religion". 
La preocupación por el discernimiento conceptual sobre emociones y estados de ánimo se advierte especialmente en los ensayos y sermones para consolar la melancolía religiosa. Muchos teólogos, especialmente anglicanos pero también algunos no conformistas como Richard Baxter, coincidían en que esta variedad de la enfermedad se caracterizaba por un trastorno fisiológico que estaba acompañado por un error teológico que llevaba a quienes la padecían a creerse condenados (Schmidt, 2007, Capítulo 4). Por eso, los pastores se esforzaban a menudo por definir apropiadamente una variedad de conceptos vinculados con estados de ánimo alterados.

Así, por ejemplo, en un sermón, Sharp establecía una distinción entre cuatro tipos de perturbaciones mentales: la melancolía que procedía de causas mundanas; la aflicción de conciencia; la escrupulosidad y la melancolía religiosa (Sharp, 1716, pp. 25-28). $\mathrm{El}$ arzobispo tenía diversos motivos para preocuparse por aclarar las diferencias. En primer lugar, era un modo de llevar certidumbre a los melancólicos de que ellos eran, en realidad, "personas muy inocentes y virtuosas" 28 (Sharp, 1716, p. 28), víctimas de una enfermedad. En segundo término, le interesaba aclarar que ese trastorno era distinto de los problemas de conciencia que afligían a los pecadores, como a los disidentes de la Iglesia establecida, a quienes había dedicado un relevante tratado en dos partes (Sharp, 1684, 1685). Finalmente, quería mostrar que el ejercicio correcto de la religión no debía conducir a la melancolía y, así, reprender al mismo tiempo a los impiadosos que carecían del "coraje para comprometerse sinceramente con ella" -según la cita al principio de este artículo-y a los entusiastas que quisieran guiar a otros por un inconducente valle de lágrimas.

Otro teólogo preocupado por establecer distinciones conceptuales acerca de emociones legítimas y patológicas era el hijo de Susanna y fundador del movimiento metodista, John Wesley. En un sermón de 1751 titulado The Wilderness State, empleaba una analogía con el paso del pueblo de Israel por el desierto para referirse al abatimiento que a menudo acontecía a los creyentes poco después de la conversión. Allí equiparaba esa "condición de desierto" con la "oscuridad" (darkness) que otros autores consideraban una instancia purificadora del alma. En contra de ellos, el metodista lo concebía como un estado patológico que se caracterizaba por la pérdida de la fe, el amor, el gozo, la paz y el poder sobre el pecado, y por eso respondía a sus adversarios:

¡Fuera esa idea absurda de que el reino de Dios está dividido contra sí mismo; que la paz de Dios y el gozo en el Espíritu Santo son obstáculos de la justicia; y que somos salvados, no por la fe, sino por la incredulidad; no por la esperanza, sino por la desesperación! (J. Wesley, 1920, pp. 261-262). ${ }^{29}$

Por cierto, Wesley no creía que toda forma de tristeza fuera espiritualmente patológica, sino solo la que conducía a la pérdida de la fe. En Heaviness Through Manifold Temptations, un sermón de 1754 que hacía referencia al anterior, hablaba sobre las aflicciones que cualquier cristiano podía atravesar durante la santificación al estar expuesto a múltiples tentaciones. Allí establecía una distinción entre la "oscuridad" y la "pesadumbre" (heaviness), un término que tomaba de 1 Pedro 1: 6, según el texto de la versión King James. El teólogo explicaba que se trataba de una traducción del griego $\lambda v \pi \eta \theta \varepsilon v \tau \varepsilon \varsigma$ (lyphthentes) que podía entenderse como "apenados", "doloridos" o "tristes", pero que la elección de heaviness daba a entender que se trataba de una tristeza profunda y prolongada (J. Wesley, 1920, p. 267).

28 "very innocent, and vertuous Persons".

29 "Away then with the idle conceit, that the kingdom of God is divided against itself; that the peace of God, and joy in the Holy Ghost, are obstructive of righteousness; and that we are saved, not by faith, but by unbelief; not by hope, but by despair!" 
Wesley no era el arzobispo de York sino que -un poco a su pesar- era el líder de un movimiento revulsivo que operaba en los márgenes de la Iglesia de Inglaterra y, por lo tanto, su intención con estos sermones era algo diferente a la de Sharp. Su objetivo era, al mismo tiempo, criticar las ideas acerca del carácter purificador de la oscuridad defendidas por algunos místicos -católicos, como San Juan de la Cruz, pero también protestantes como William Law, en sus escritos más tardíos, luego de conocer la obra de Jacob Böhme- y defender su teología de las acusaciones de entusiasmo que recaían a menudo sobre los metodistas.

Esta brevísima síntesis permite ilustrar que diversos términos -algunos más específicos como despair o melancholy, y otros más metafóricos como darkness o heaviness-adquirían sentidos específicos en el marco de operaciones polémicas concretas. En ellas se disputaban, además de la legitimidad particular de cada denominación, confesión o movimiento religioso, un conjunto de valores, normas y representaciones acerca de la experiencia afectiva de los cristianos. Por lo tanto, el análisis histórico de las especificidades de las emociones protestantes no puede avanzar a partir de una lectura ingenua de las fuentes.

\section{Conclusión}

En su diario, Susanna Wesley se quejaba de cómo la parte profana del mundo acusaba a la religión de melancolía. En las páginas anteriores, se mostró que existe una persistente tradición en la literatura sociológica e histórica que ha pretendido representar el papel del profano en su estudio del protestantismo y le atribuyó a este movimiento religioso la doble facultad de ser un factor dinámico de modernización y la fuente de un sufrimiento psicológico profundo, incluso patológico.

El "mito del calvinista desesperado" fue, según Ryrie, un invento de Max Weber. En rigor, lo que el alemán hizo fue darle entidad científica a una afirmación polémica que distintos grupos religiosos habían hecho sobre otros para disputar su legitimidad. Como parte de su tesis sobre la modernización y el desencantamiento del mundo, las ideas de Weber acerca de la aflicción protestante ejercieron una influencia significativa en la historiografía inglesa. Sin embargo, solo algunos autores convirtieron a este aspecto en un elemento central de sus explicaciones acerca de la cultura protestante inglesa. Además, fue como una radicalización de los argumentos de Weber, e incluso en contra suyo, que autores como Stachniewski, Rubin y Simpson desarrollaron la idea de una cultura patológica que producía como una experiencia típica y deseable la desesperación religiosa.

En las últimas décadas, los historiadores de las emociones propusieron interpretaciones más complejas y fructíferas acerca del papel de la aflicción de los protestantes ingleses. Estas permiten huir de las simplificaciones y las lecturas encerradas en viejas polémicas teológicas, pero sin eludir el estudio de la relación específica de aquella cultura religiosa con la angustia, la tristeza, el miedo, la melancolía o la desesperación.

Finalmente, este artículo buscó enfatizar la relevancia de la dimensión polémica de los discursos religiosos acerca de las emociones. La facilidad con la que, en el fragor de la lucha, "la parte profana del mundo" y "la religión" intercambian posiciones, hace necesario mantener la guardia alta. Por eso, el estudio de la aflicción protestante requiere un cuidadoso análisis contextual y conceptual de las fuentes empleadas. 


\section{Q Bibliografía}

" Addison, J., \& Steele, R. (1806). The Spectator (A. Chalmers, Ed.; Vol. 7). Londres: J. Johnson et al.

"Asprem, E. (2014). The Problem of Disenchantment. Scientific Naturalism and Esoteric Discourse, 1900-1939. Albany: State University of New York Press.

》 Bartra, R. (2018 [2004]). El spleen del capitalismo: Weber y la ética pagana. En El duelo de los ángeles: Locura sublime, tedio y melancolía en el pensamiento moderno (pp. 36-60). México: Fondo de Cultura Económica.

» Bell, M. (2014). Melancholia. The Western Malady. Cambridge: Cambridge University Press.

» Benjamin, W. (2012 [1925]). Origen del Trauerspiel alemán (Trad. Pivetta, C.). Buenos Aires: Gorla.

"Bjerg, M. (2019). Una genealogía de la historia de las emociones. Quinto Sol, 23(1), 1-20.

" Boddice, R. (2018). The History of Emotions. Manchester: Manchester University Press.

» Bunyan, J. (1996 [1678]). The Pilgrim's Progress. Chatham: Wordsworth.

" Burton, R. (2001 [1621]). The Anatomy of Melancholy. Nueva York: New York Review of Books.

»Clarke, E. (1886). Susanna Wesley. Londres: W. H. Allen \& co.

"Collinson, P. (2003). Elizabethans. Cambridge: Cambridge University Press.

"Cooper, T. (2011). John Owen, Richard Baxter and the Formation of Nonconformity. Farnham: Ashgate.

»Crouzet, D. (2001). Calvino. Barcelona: Ariel.

» Cummins, S., \& Stille, M. (2021). Religious Emotions and Emotions in Religion: The Case of Sermons. Journal of Religious History, 45(1), 3-24. https://doi.org/10.1111/1467-9809.12726

》Dallimore, A. (1993). Susanna Wesley. The Mother of John and Charles Wesley. Grand Rapids: Baker Book House.

»Delumeau, J. (1991). Sin and Fear: The Emergence of a Western Guilt Culture, 13th-18th Centuries. New York: St. Martin's Press.

»Delumeau, J. (2006). Lo mandado y lo vivido. En El cristianismo del futuro (pp. 331-361). Bilbao: Ediciones Mensajero.

»Delumeau, J. (2017). El miedo en occidente. Barcelona: Taurus.

» Dixon, T. (2012). "Emotion": The History of a Keyword in Crisis. Emotion Review, 4(4), 338-344.

"Dowden, E. (1910). Puritan and Anglican. Studies in Literature (3.a ed.). Londres: Kegan Paul, Trench, Trübner \& Co. Ltd.

"Dunan-Page, A. (2006). Grace Overwhelming: John Bunyan, the Pilgrim's Progress and the Extremes of the Baptist Mind. Berna: Peter Lang.

"Durkheim, É. (2002 [1897]). Suicide: A Study in Sociology (Ed. Simpson, G.). LondresNueva York: Routledge.

» Frevert, U., Scheer, M., Schmidt, A., Eitler, P., Hitzer, B., Verheyen, N., Gammerl, B., Bai- 
ley, C., \& Pernau, M. (2014). Emotional Lexicons. Continuity and Change in the Vocabulary of Feeling 1700-2000. Oxford: Oxford University Press.

" Fromm, E. (1973 [1941]). El miedo a la libertad. Buenos Aires: Paidós.

" Gauchet, M. (2005). El desencantamiento del mundo. Una historia política de la religión (Trad. Molina, E.). Madrid: Trotta / Universidad de Granada.

» Gil Villegas M., F. (2013). Max Weber y la guerra académica de los cien años. La polémica en torno a La ética protestante y el espíritu del capitalismo (1905-2012). México: Fondo de Cultura Económica.

" Ginzburg, C. (2012). Our Words, and Theirs: A Reflection on the Historian's Craft, Today. En S. Fellman \& M. Rahikainen (Eds.), Historical Knowledge: In Quest of Theory, Method and Evidence (97-119). Cambridge: Cambridge Scholars Publishing.

" Gowland, A. (2006a). The Worlds of Renaissance Melancholy. Robert Burton in Context. Cambridge: Cambridge University Press.

" Gowland, A. (2006b). The Problem of Early Modern Melancholy. Past \& Present, 191(1), 77-120.

» Haller, W. (1938). The Rise of Puritanism. Nueva York y Londres: Columbia University Press.

»Harmon, R. L. (1968). Susanna, Mother of the Wesleys. Nashville: Abingdon Press.

» Harrison, G. E. (1938). Son To Susanna: The Private Life OfJohn Wesley. Nashville: Cokesbury Press.

" Heyd, M. (1981). The Reaction to Enthusiasm in the Seventeenth Century: Towards an Integrative Approach. The Journal of Modern History, 53(2), 258-280.

" Heyd, M. (1995). "Be Sober and Reasonable». The Critique of Enthusiasm in the Seventeenth and Early Eighteenth Centuries. Leiden-Nueva York-Colonia: Brill.

» Hill, C. (1961). Protestantism and the Rise of Capitalism. En F. J. Fisher (Ed.), Essays in the Economic and Social History of Tudor and Stuart England (15-39). Cambridge: Cambridge University Press.

» Hill, C. (1975). The World Turned Upside Down. Radical Ideas During the English Revolution. Harmondsworth: Penguin.

» Hill, C. (1990 [1970]). God's Englishman. Oliver Cromwell and the English Revolution. Londres: Penguin.

" Hill, C. (1993). Review: The Persecutory Imagination: English Puritanism and the Literature of Religious Despair. Literature \& History, 2(2), 96-98.

》 Hill, C. (2016 [1988]). A Turbulent, Seditious, and Factious People. John Bunyan and His Church, 1628-1688. Londres: Verso.

" Hodgkin, K. (2007). Madness in Seventeenth-Century Autobiography. Basingstoke: Palgrave Macmillan.

" Jason A., J.-S. (2017). The Myth of Disenchantment. Magic, Modernity, and the Birth of the Human Sciences. Chicago: The University of Chicago Press.

" Johnstone, N. (2006). The Devil and Demonism in Early Modern England. Cambridge: Cambridge University Press.

» Kaufman, P. I. (1996). Prayer, Despair, and Drama: Elizabethan Introspection. Urbana: University of Illinois Press.

» Kirk, J. (1865). The Mother of the Wesleys: A Biography. Cincinnati: Poe and Hitchcock. 
» Kleinman, A. (1988). Rethinking Psychiatry: From Cultural Category to Personal Experience. Nueva York: The Free Press.

"Kleinman, A. (2020). The Illness Narratives: Suffering, Healing, And The Human Condition. Nueva York: Basic Books.

» Knox, R. A. (1950). Enthusiasm: A Chapter in the History of Religion, with Special Reference to the XVII and XVIII Centuries. Oxford: Oxford University Press.

» Laborie, L. (2015). Enlightening Enthusiasm: Prophecy and Religious Experience in Early Eighteenth-century England. Oxford: Oxford University Press.

» Law, W. (1729). A Serious Call to a Devout and Holy Life. Adapted to the State and Condition of All Orders of Christians. Londres: William Innys.

» Lecky, W. E. H. (1921 [1892]). A History Of England In The Eighteenth Century: Vol. III. Londres: Longmans, Green and Co.

» Lewis, C. S. (1954). English Literature in the Sixteenth Century Excluding Drama. Oxford: Clarendon Press.

» Lim, P. C.-H. (2004). In Pursuit of Purity, Unity, and Liberty: Richard Baxter's Puritan Ecclesiology in Its Seventeenth-Century Context. Leiden-Boston: Brill.

» Luther, M. (2002). D. Martin Luthers Werke (U. Köpf, H. Junghans, \& K. Stackmann, Eds.; Vol. 11). Weimar: Böhlaus.

» Lutz, C. A. (1998). Unnatural Emotions. Everyday Sentiments on a Micronesian Atoll and their Change to Western Theory. Chicago: The University of Chicago Press.

» Lutz, C. A. \& Abu-Lughod, L. (1990). Language and the Politics of Emotion. Cambridge: Cambridge University Press.

» MacDonald, M. (1992). The Fearefull Estate of Francis Spira: Narrative, Identity, and Emotion in Early Modern England. Journal of British Studies, 31(1), 32-61.

" Méndez, A. (2021). Miedo, epidemia y brujería. Apuntes sobre la comunidad emocional de un clérigo puritano inglés (s. XVI y XVII). XI Jornadas de Historia Moderna y Contemporánea, Bahía Blanca.

"Newton, J. A. (1968). Susanna Wesley and the Puritan Tradition in Methodism. Londres: Epworth Press.

» Oatley, K. (2004). Emotions: A Brief History. Oxford: Blackwell.

» Parish, H. (Ed.). (2015). "Disenchantment» of Europe? En Superstition and Magic in Early Modern Europe. A Reader (pp. 307-385). Londres: Bloomsbury.

» Pernau, M., \& Rajamani, I. (2016). Emotional Translations: Conceptual History Beyond Language. History and Theory, 55(1), 46-65.

» Plamper, J. (2012). The History of Emotions: An Introduction. Oxford: Oxford University Press.

» Pocock, J. G. A. (1997). Enthusiasm: The Antiself of Enlightenment. Huntington Library Quarterly, 6o(1/2), 7-28.

» Rack, H. D. (2004). Wesley, Samuel (bap. 1662, d. 1735). Oxford: Oxford University Press.

» Reddy, W. M. (2001). The Navigation of Feeling. A Framework for the History of Emotions. Cambridge: Cambridge University Press.

" Rosaldo, M. Z. (1980). Knowledge and Passion: Ilongot and Notions of Self and Social Life. Cambridge: Cambridge University Press.

» Rosenwein, B. H. (2006). Emotional Communities in the Early Middle Ages. Ithaca: Cornell University Press. 
» Rosenwein, B. H. (2016). Generations of Feeling. A History of Emotions, 600-1700. Cambridge: Cambridge University Press.

» Rosenwein, B. H. (2018). Were Puritan Emotions Gendered? (New England, mid-16oos). Clio. Women, Gender, History, 47, 67-88.

» Rubin, J. H. (1994). Religious Melancholy and Protestant Experience in America. Oxford: Oxford University Press.

» Ryrie, A. (2013). Being Protestant in Reformation Britain. Oxford: Oxford University Press.

» Ryrie, A.\& Schwanda, T. (Eds.). (2016). Puritanism and Emotion in the Early Modern World. Basingstoke: Palgrave MacMillan.

» Scaer, D. P. (1983). The Concept of Anfechtung in Luther's Thought. Concordia Theological Quarterly, 47(1), 15-30.

» Scheer, M. (2020). Enthusiasm. Emotional Practices of Conviction in Modern Germany. Oxford: Oxford University Press.

"Schmidt, J. (2007). Melancholy and the Care of the Soul. Religion, Moral Philosophy and Madness in Early Modern England. Hampshire: Ashgate.

»Scribner, R. W. (1993). The Reformation, Popular Magic, and the «Disenchantment of the World». The Journal of Interdisciplinary History, 23(3), 475-494. https://doi. org/10.2307/206099.

»Shapin, S. \& Schaffer, S. (1985). Leviathan and the Air-Pump. Hobbes, Boyle, and the Experimental Life. Princeton: Princeton University Press.

»Sharp, J. (1684). A Discourse Concerning Conscience; Wherein An Account is Given of the Nature, and Rule, and Obligation of It. And the Case of Those Who Separate from the Communion of the Church of England as by Law Established, Upon this Pretence, that it is Against their Conscience to Joyn It, is Stated and Discussed. Londres: Walter Kettilby.

"Sharp, J. (1685). A Discourse of Conscience. Concerning a Doubting Conscience. Londres: Walter Kettilby.

» Sharp, J. (1716). Sixteen Casuistical Sermons Preached on Several Occasions. Londres: Walter Kettilby.

» Simons, R. C. \& Hughes, C. C. (2012 [1985]). The Culture-Bound Syndromes: Folk Illnesses of Psychiatric and Anthropological Interest. Dordrecht: D. Reidel.

» Simons, R. C., \& Hughes, C. C. (1993). Culture-Bound Syndromes. En A. Gaw (Ed.), Culture, Ethnicity, and Mental Illness (75-93). Washington D. C.-Londres: American Psychiatric Press.

»Simpson, J. (2019). Permanent Revolution. The Reformation and the Illiberal Roots of Liberalism. Cambridge, MA-Londres: Harvard University Press.

»Skinner, Q. (2002). Visions of Politics. Regarding Method (Vol. 1).

» Stachniewski, J. (1991). The Persecutory Imagination: English Puritanism and the Literature of Religious Despair. Oxford: Clarendon Press.

»Starkie, A. (2002). William Law and Cambridge Jacobitism, 1713-16. Historical Research, 75(190), 448-467. https://doi.org/10.1111/1468-2281.0016o.

»Sullivan, E. (2016). Beyond Melancholy: Sadness and Selfhood in Renaissance England. Oxford: Oxford University Press.

» Tawney, R. H. (1937). Religion and the Rise OfCapitalism (2.a ed.). West Drayton: Penguin.

» Thomas, K. (1991 [1971]). Religion and the Decline of Magic. Studies in Popular Beliefs in 
Sixteenth and Seventeenth-Century England. Londres: Penguin.

» Thomas, K. (2009). The Ends of Life. Roads to Fulfilment in Early Modern England. OxfordNueva York: Oxford University Press.

» Thomas, K. (2020). Calvinism's Discontents: Does Liberalism Have Its Roots in the Illiberal Upheavals of the English Reformation? The Nation. https://www.thenation.com/ article/culture/james-simpson-permanent-revolution-review/.

» Thompson, E. P. (1966). The Making of the English Working Class. Londres: Vintage.

» Torpy, A. A. (2009). The Prevenient Piety of Samuel Wesley, Sr. Lanham-Toronto-Plymouth: Scarecrow Press.

» Trevor-Roper, H. R. (1959). The General Crisis of the 17th Century. Past and Present, 16(1), $31-64$.

» Tyerman, L. (1866). Life and Times of the Rev. Samuel Wesley, M.A. Londres: Simpkin, Marshall \& Co.

"Walker, D. (2016). Piety and the Politics of Anxiety in Nonconformist Writing of the Later Stuart Period. En A. Ryrie \& T. Schwanda (Eds.), Puritanism and Emotion in the Early Modern World (144-165). Basingstoke: Palgrave MacMillan.

»Walker, D. P. (1964). The Decline of Hell. Seventeenth-Century Discussions of Eternal Torment. Londres: Routledge \& Kegan Paul.

»Wallace, C. (2010). Wesley [née Annesley], Susanna (1669-1742), theological writer and educator. En Oxford Dictionary of National Biography. Oxford: Oxford University Press. https://doi.org/10.1093/ref:odnb/66878

»Walzer, M. (1963). Puritanism as a Revolutionary Ideology. History and Theory, 3(1), 59-90. https://doi.org/10.2307/2504304

»Walzer, M. (1965). The Revolution of the Saints. A Study in the Origins of the Radical Politics. Cambridge, MA-Londres: Harvard University Press.

»Weber, M. (1992). Wissenschaft als Beruf. En Gesamtausgabe: Vol. XVII (71-111). Tubinga: J. C. B. Mohr.

»Weber, M. (2004 [1905]). La ética protestante y el espíritu del capitalismo. Buenos Aires: Andrómeda.

»Weber, M. (2004). Science as a Vocation. En D. Owen \& T. B. Strong (Eds.), The Vocation Lectures (Trad. Livingstone, R.) (1-31). Indianapolis-Cambridge: Hackett Publishing.

»Weber, M. (2016). Die protestantische Ethik und der „Geist“ des Kapitalismus. Wiesbaden: Springer.

»Wesley, J. (1920). Wesley’s Standard Sermons: Vol. II. Nashville: Publishing House M. E. Church.

»Wesley, S. (1997). Susanna Wesley: The Complete Writings (Ed. Wallace, C. Jr.). Oxford: Oxford University Press.

»Williamson, G. (1933). The Restoration Revolt Against Enthusiasm. Studies in Philology, 30(4), 571-603.

»Zafirovski, M. (2007). The Protestant Ethic and the Spirit of Authoritarianism. Puritanism, Democracy, and Society. Nueva York: Springer.

» Žižek, S. (2009). The Fear of Four Words: A Modest Plea for the Hegelian Reading of Christianity. En C. Davis (Ed.), The Monstrosity of Christ: Paradox or Dialectic? (24-109). Cambridge, MA-Londres: The MIT Press. 